INPLASY

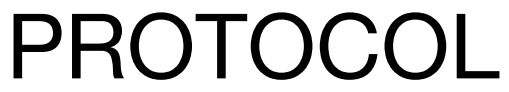

To cite: Han et al. Effect of Acupuncture on Glucose and Lipid Metabolism in Obese Type 2 Diabetes: A Systematic Review and Meta-Analysis. Inplasy protocol 202130087. doi:

10.37766/inplasy2021.3.0087

Received: 22 March 2021

Published: 23 March 2021

Corresponding author: Mingjun Liu

liumingjun123@hotmail.com

Author Affiliation:

Changchun University of

Chinese Medicine,

Changchun, Jilin Province, PR

China

Support: NSFC.

Review Stage at time of this submission: Preliminary searches.

Conflicts of interest: None declared.

\section{Effect of Acupuncture on Glucose and Lipid Metabolism in Obese Type 2 Diabetes: A Systematic Review and Meta-Analysis}

Han, X1; Lu, Z²; Meng, M³; Wang, H; Ting, P5; Tianjiao, G6; Liu, M

Review question / Objective: It has been unclear whether acupuncture therapy improves clinical outcomes in obese type 2 diabetic patients. This meta-analysis aimed to summarize the effect of acupuncture on assess the impact of supplemental probiotics on fasting plasma glucose (FPG), glycated hemoglobin (HbA1c), fasting insulin, and lipid profile, from clinical trials.

Condition being studied: Obesity and type 2 diabetes mellitus (T2D) are global pandemics. Worldwide, the prevalence of obesity has nearly tripled since 1975 and the prevalence of T2D has almost doubled since 1980. In general, the most important comorbidity of obesity is type 2 diabetes mellitus(T2DM), as it plays a central role in the development of other comorbidities and further aggravates the metabolic syndrome. Obesity is a major risk factor for the development of T2DM, as the associated chronic, low-grade, sterile inflammation contributes to both insulin resistance and b-cell failure. In the United Kingdom (UK), of the $\mathbf{3 . 2}$ million people with diabetes, an estimated $80 \%$ to $85 \%$ are overweight or obese, in whom weight gain could be potentially detrimental.

INPLASY registration number: This protocol was registered with the International Platform of Registered Systematic Review and Meta-Analysis Protocols (INPLASY) on 23 March 2021 and was last updated on 23 March 2021 (registration number INPLASY202130087).

\section{INTRODUCTION}

Review question / Objective: It has been unclear whether acupuncture therapy improves clinical outcomes in obese type 2 diabetic patients. This meta-analysis aimed to summarize the effect of acupuncture on assess the impact of supplemental probiotics on fasting plasma glucose (FPG), glycated hemoglobin (HbA1c), fasting insulin, and lipid profile, from clinical trials.

Condition being studied: Obesity and type 2 diabetes mellitus (T2D) are global pandemics. Worldwide, the prevalence of 
obesity has nearly tripled since 1975 and the prevalence of T2D has almost doubled since 1980. In general, the most important comorbidity of obesity is type 2 diabetes mellitus(T2DM), as it plays a central role in the development of other comorbidities and further aggravates the metabolic syndrome. Obesity is a major risk factor for the development of T2DM, as the associated chronic, low-grade, sterile inflammation contributes to both insulin resistance and $b$-cell failure. In the United Kingdom (UK), of the 3.2 million people with diabetes, an estimated $80 \%$ to $85 \%$ are overweight or obese, in whom weight gain could be potentially detrimental.

\section{METHODS}

Participant or population: Obese type 2 diabetic patients.

Intervention: acupuncture was the main intervention (e.g., body acupuncture, abdominal acupuncture and electropuncture).

Comparator: Patients in the control group were treated with sham acupuncture, placebo, or hypoglycemic drugs.

Study designs to be included: Randomized controlled clinical trials and nonrandomized controlled trials will be included.

Eligibility criteria: Participants with standards of body mass index (BMI) $\geq 24$ $\mathrm{kg} / \mathrm{m} 2$ which are diagnosed withT2DM will be included. The diagnosis of T2DM is in accordance with who's diagnostic criteria for diabetes in 1999. Mean-while, the participants who are included have no limitation of age, sex, region, citizenship, and nationality. Cases related to serious diseases, pregnancy, and drug-induced obesity are excluded.

Information sources: We will electronically search PubMed, Medline, Embase, Web of Science, the Cochrane Central Register of Controlled Trials, China National Knowledge Infrastructure, Chinese Biomedical Literature Database, Chinese
Scientific Journal Database, and Wan-Fang Database from the date of creation to December 2022. In addition, we will manually retrieve other resources including the reference lists of identified publications, conference articles, and gray literature.

Main outcome(s): (1)fasting plasma glucose (FPG), (2)glycated hemoglobin (HbA1c), (3)fasting insulin, (4) lipid profile.

Additional outcome(s): (1) weight loss (kg), (2) homeostatic model assessment of insulin resistance (HOMA-IR) level, (3)CRP level , (4) HOMA-IR, (5) dropout, (6) side effects, and (7) rebound.

Quality assessment / Risk of bias analysis: The literature quality of this study was evaluated by the bias risk table proposed by Cochrane collaborative network. The risk table includes 6 items: random sequence generation mode, whether to use allocation concealment, whether to blind the subjects and intervention providers, whether to blind the results evaluators, whether the results data are complete, whether to select the results report and other bias sources. The criteria used to assess the risk of bias are "low risk," "high risk," and "unclear." In this process, 2 evaluators independently evaluate the methodological quality. In case of disagreement, the third author would be intervened.

Strategy of data synthesis: The terms for searching were: ('acupuncture OR body acupuncture OR abdominal acupuncture OR electropuncture) AND ('supple intervention') AND ('type 2 diabetes mellitus') AND ('obese') AND (glucose metabolism OR Lipid metabolism) AND (randomized OR blind OR placebo OR meta-analysis). We also attempted to contact the investigators if their clinical end-points were not reported.

Subgroup analysis: We will consider subgroups such as jurisdiction, clinic type, and location(rural/urban). 
Sensitivity analysis: The literature quality of this study was evaluated by the bias risk table proposed by Cochrane collaborative network. The risk table includes 6 items: random sequence generation mode, whether to use allocation concealment, whether to blind the subjects and intervention providers, whether to blind the results evaluators, whether the results data are complete, whether to select the results report and other bias sources. The criteria used to assess the risk of bias are "low risk," "high risk," and "unclear." In this process, 2 evaluators independently evaluate the methodological quality. In case of disagreement, the third author would be intervened.

Country(ies) involved: American, China.

Keywords: acupuncture; type 2 diabetes; obese; meta-analysis.

Contributions of each author:

Author 1 - Yiran Han - The author drafted the manuscript.

Email: miaran0910@163.com

Author 2 - Zeyuan Lu - The author provided statistical expertise.

Author 3 - Meng Meng.

Author 4 - Heran Wang.

Author 5 - Pan Ting.

Author 6 - Gao Tianjiao.

Author 7 - Mingjun Liu. 